\title{
Stabilized semi-implicit spectral deferred correction methods for Allen-Cahn and Cahn-Hilliard equations
}

\author{
Fei Liu ${ }^{\mathrm{a}}$ and Jie Shen ${ }^{\mathrm{b}, \mathrm{c} * \dagger}$
}

\section{Communicated by Q. Wang}

Stabilized semi-implicit spectral deferred correction methods are constructed for the time discretization of Allen-Cahn and Cahn-Hilliard equations. These methods are unconditionally stable, lead to simple linear system to solve at each iteration, and can achieve high-order time accuracy with a few iterations in each time step. Ample numerical results are presented to demonstrate the effectiveness of the stabilized semi-implicit spectral deferred correction methods for solving the Allen-Cahn and Cahn-Hilliard equations. Copyright $\odot 2013$ John Wiley \& Sons, Ltd.

Keywords: spectral deferred correction; spectral Galerkin method; method of lines; Allen-Cahn and Cahn-Hilliard equations

\section{Introduction}

We consider in this paper the numerical approximation of the Allen-Cahn equation

$$
\partial_{t} u=\gamma\left[\Delta u-\frac{1}{\varepsilon^{2}} f(u)\right],(x, t) \in \Omega \times(0, T],
$$

and the Cahn-Hilliard equation

$$
\partial_{t} u=\gamma \Delta\left[-\Delta u+\frac{1}{\varepsilon^{2}} f(u)\right],(x, t) \in \Omega \times(0, T],
$$

with the initial condition $\left.u\right|_{t=0}=u_{0}$ and a suitable set of boundary conditions, for example, periodic boundary conditions or homogeneous Neumann boundary conditions

$$
\left.\frac{\partial u}{\partial n}\right|_{\partial \Omega}=0
$$

for (1.1) and

$$
\left.\frac{\partial u}{\partial n}\right|_{\partial \Omega}=0,\left.\frac{\partial \Delta u}{\partial n}\right|_{\partial \Omega}=0
$$

for (1.2). In the previous equation, $f(u)=\left(u^{2}-1\right) u=\mathcal{F}^{\prime}(u)$ where $\mathcal{F}(u)=\frac{1}{4}\left(u^{2}-1\right)^{2}$ is the usual double-well potential, $\Omega$ is a bounded domain, and $n$ is the outward normal. We have introduced a parameter $\varepsilon$ explicitly in (1.1) and (1.2) to highlight additional difficulties that may arise when $\varepsilon \ll 1$ as in interfacial problems.

The Allen-Cahn Equation (1.1) was originally introduced in [1] to describe the motion of anti-phase boundaries in crystalline solids, whereas the Cahn-Hilliard Equation (1.2) was introduced by [2] to describe the complicated phase separation and coarsening phenomena in a solid. These equations have been used extensively in materials science as model for phase transition, pattern formation, and

\footnotetext{
${ }^{a}$ School of Mathematics and Statistics, Huazhong University of Science and Technology, Wuhan, China

${ }^{b}$ School of Mathematical Science, Xiamen University, Xiamen, China

'Department of Mathematics, Purdue University, West Lafayette, IN 47907-1957, USA 
such, and have been the subject of extensive mathematical and numerical studies (see, e.g.,[3-6] and the references therein). It is still a challenge to construct efficient high-order, unconditionally energy stable schemes that are robust with small $\varepsilon$.

Although an enormous body of work have been devoted to developing high-order or spectrally accurate schemes for spatial variables (cf. [7-11] and the references therein), much less attention has been paid to developing high-order or spectrally accurate schemes in time, some efforts in this direction include [12-19]. Although in principle, one can apply a spectral-collocation or spectral Galerkin method to the time variable, a fundamental difficulty is how to solve the resultant nonlinear system efficiently.

Note that it is certainly not a good idea to treat the whole time interval with one spectral element, as it will lead to very large nonlinear systems that are hard to store and solve. Instead, we should adopt the $h-p$ finite element (or spectral element) strategy in time to divide the whole time interval into a sequence of smaller intervals $\left[s_{i}, s_{i+1}\right], i=0,1, \ldots, M-1$ and construct a spectrally accurate time marching scheme from time $s_{i}$ to $s_{i+1}$. We note that for the $h-p$ method applied to the spatial variables, it is not possible to decouple the computation element-wise; however, the fact that the time variable has a direction makes the subinterval-wise decoupling possible in time.

The crucial issue is how to solve the nonlinear systems at each time interval stably and efficiently. The advent of spectral/Krylov deferred correction (SDC/KDC) method [14] provided a good starting point. We shall describe later in the text several strategies that will allow us to construct high-order or even spectrally accurate schemes in time for a class of partial differential equations in fluid mechanics and materials science.

To use the SDC/KDC approach, the main difficulty is to construct an efficient basic scheme that is unconditionally energy stable. One option is to use a convex splitting scheme [20] which is unconditionally energy stable but leads to a nonlinear system to solve at each time step. Instead, we propose to use a stabilized semi-implicit scheme (cf. [21]) that is unconditionally stable and leads to a system of Poisson type equations that can be solved very efficiently. Thus, this approach will allow us to use large time steps with high-order accuracy in time, which is otherwise very difficult to achieve.

Although we only consider the Allen-Cahn and Cahn-Hilliard equations in this paper, it is clear that this approach can be applied to more general gradient flows, including the equations for thin film epitaxy (cf. [22-24]) and anisotropic Cahn-Hilliard equations (cf. [25-27]). Moreover, this approach is an important component for developing high-order stable schemes for phase-field models of the multiphase flows (cf. for instance [28] and the references therein).

The remainder of the paper is organized as follows. In the next section, we briefly describe the SDC and the semi-implicit SDC (SISDC) methods. In Section 3, we construct the stabilized semi-implicit SDC (SSISDC) scheme for the Allen-Cahn and Cahn-Hilliard equations. We present ample numerical results to demonstrate the high-order (in time) accuracy of the SSISDC method in Section 4, followed by some concluding remarks.

\section{SDC and SISDC Schemes for system of ODEs}

The SDC method was first introduced by Dutt, Greengard, and Rokhlin in [14] to construct high-order stable methods for solving ODEs. The basic idea of the SDC method is to replace the original ODE by the corresponding Picard integral equation and discretize it on a sequence of large time intervals by using a Legendre-Gauss-type quadrature. The resultant system is first solved approximately by using a simple time marching numerical method and correct the solution to higher-order accuracy by solving a sequence of correction equations on the same Gauss-type grid with a simple time marching scheme (such as the Euler method).

For the readers' convenience, we describe later in the text the basic SDC method in some detail. Consider the following system of ODEs:

$$
\begin{aligned}
& \phi^{\prime}(t)=F(t, \phi(t)), \quad t \in[0, T] \\
& \phi(0)=\phi_{0}
\end{aligned}
$$

where $\phi(t), \phi_{0} \in \mathbb{C}^{n}$ and $F: \mathbb{R} \times \mathbb{C}^{n} \rightarrow \mathbb{C}^{n}$. We assume that $F$ is sufficiently smooth such that the problem (2.1) is well posed.

We subdivide the time interval $[0, T]$ in $K$ non-overlapping intervals: $[0, T]=\cup_{j=0}^{K-1}\left[t_{j}, t_{j+1}\right]$. We shall describe later in the text the SDC method that will be used to advance from $t_{j}$ to $t_{j+1}$. To simplify the notation, we shall denote the interval $\left[t_{j}, t_{j+1}\right]$ by the generic interval $[a, b]$.

\subsection{Picard integral equation}

The first key idea of the SDC method is to rewrite (2.1) in the form of Picard integral equation. Integrating (2.1) from $a$ to $t$, we obtain the equivalent Picard integral equation

$$
\phi(t)=\phi_{a}+\int_{a}^{t} F(\tau, \phi(\tau)) d \tau .
$$

Given an initial approximate solution $\phi^{0}(t)$ to (2.2), we denote the error by $\delta(t)=\phi(t)-\phi^{0}(t)$. Another measure for the quality of the approximation is given by the residual function

$$
\epsilon\left(t, \phi^{0}\right)=\phi_{a}+\int_{a}^{t} F\left(\tau, \phi^{0}(\tau)\right) d \tau-\phi^{0}(t) .
$$


Because $\phi(t)=\phi^{0}(t)+\delta(t)$, we can rewrite (2.2) as

$$
\phi^{0}(t)+\delta(t)=\phi_{a}+\int_{a}^{t} F\left(\tau, \phi^{0}(\tau)+\delta(\tau)\right) d \tau .
$$

Combining the previously mentioned equation with (2.3), we derive

$$
\delta(t)=\int_{a}^{t}\left[F\left(\tau, \phi^{0}(\tau)+\delta(\tau)\right)-F\left(\tau, \phi^{0}(\tau)\right)\right] d \tau+\epsilon\left(t, \phi^{0}\right),
$$

which is referred to as the correction equation.

\subsection{The SDC method based on the Euler method}

We denote the $p$ Legendre-Gauss-Radau Ila nodes (including the right end point) on the interval $[-1,1]$ by $r_{1}, r_{2}, \ldots, r_{p}$, which is a strictly increasing sequence of points, and denote by $s_{1}, s_{2}, \ldots, s_{p}$ the $p$ Legendre-Gauss-Radau lla nodes on the interval $[a, b]$, given by the formula

$$
s_{i}=\frac{b-a}{2} r_{i}+\frac{b+a}{2}
$$

We then divide the interval $[a, b]$ into $p$ subintervals $\left[s_{i}, s_{i+1}\right]$ by the Legendre-Gauss-Radau lla points $a=s_{0}<s_{1}<s_{2}<\ldots<s_{p}=b$. The subinterval $\left[s_{i}, s_{i+1}\right]$ is referred to as a substep.

An approximate solution $\phi^{0}\left(s_{i}\right)$ can be obtained by using the forward Euler method or the backward Euler method. For instance, using the notation $\phi_{i}^{k}=\phi^{k}\left(s_{i}\right)$ (likewise for $\delta_{i}^{k}$ and $\epsilon_{i}\left(\phi^{k}\right)$ ), the backward Euler scheme for (2.1) is given by the formula

$$
\phi_{i+1}^{0}=\phi_{i}^{0}+\delta t_{i} \cdot F\left(s_{i+1}, \phi_{i+1}^{0}\right), \delta t_{i}=s_{i+1}-s_{i},
$$

whereas the forward Euler scheme is

$$
\phi_{i+1}^{0}=\phi_{i}^{0}+\delta t_{i} \cdot F\left(s_{i}, \phi_{i}^{0}\right), \delta t_{i}=s_{i+1}-s_{i}
$$

for $i=0, \ldots, p-1$.

To compute a sequence of corrections $\delta_{i}^{k}$ by discretizing Equation (2.4) to obtain an increasingly accurate approximate solution $\phi^{k+1}=\phi^{k}+\delta^{k}$, the residual function $\epsilon\left(s, \phi^{k}(s)\right)$ is approximated using the spectral integration matrix

$$
\vec{\epsilon}=\vec{\phi}_{a}+\Delta t A \vec{F}-\vec{\phi}^{k}
$$

where $A$ is the spectral integration matrix [14], which was discussed in detail in [29].

We shall use the correction Equation (2.4) to construct a sequence of corrections $\left\{\delta^{k}\right\}$ and new approximations $\left\{\phi^{k}\right\}$. By using the backward Euler scheme to the correction Equation (2.4), we obtain

$$
\delta_{i+1}^{k}=\delta_{i}^{k}+\delta t_{i}\left[F\left(s_{i+1}, \phi_{i+1}^{k}+\delta_{i+1}^{k}\right)-F\left(s_{i+1}, \phi_{i+1}^{k}\right)\right]+\epsilon_{i+1}\left(\phi^{k}\right)-\epsilon_{i}\left(\phi^{k}\right) .
$$

On the other hand, the forward Euler scheme leads to

$$
\delta_{i+1}^{k}=\delta_{i}^{k}+\delta t_{i}\left[F\left(s_{i}, \phi_{i}^{k}+\delta_{i}^{k}\right)-F\left(s_{i}, \phi_{i}^{k}\right)\right]+\epsilon_{i+1}\left(\phi^{k}\right)-\epsilon_{i}\left(\phi^{k}\right) .
$$

Let us denote

$$
l_{i}^{i+1}\left(\phi^{k}\right)=\int_{s_{i}}^{s_{i+1}} F\left(t, \phi^{k}(t)\right) d t
$$

We then derive from (2.3) that

$$
l_{i}^{i+1}\left(\phi^{k}\right)=\epsilon_{i+1}\left(\phi^{k}\right)-\epsilon_{i}\left(\phi^{k}\right)+\phi_{i+1}^{k}-\phi_{i}^{k} .
$$

Because $\phi_{i+1}^{k}+\delta_{i+1}^{k}=\phi_{i+1}^{k+1}$, substituting (2.10) into Equation (2.8), we obtain the equation

$$
\phi_{i+1}^{k+1}=\phi_{i}^{k+1}+\delta t_{i}\left[F\left(s_{i+1}, \phi_{i+1}^{k+1}\right)-F\left(s_{i+1}, \phi_{i+1}^{k}\right)\right]+l_{i}^{i+1}\left(\phi^{k}\right) .
$$

On the other hand, substituting (2.10) into Equation (2.9), we obtain the equation

$$
\phi_{i+1}^{k+1}=\phi_{i}^{k+1}+\delta t_{i}\left[F\left(s_{i}, \phi_{i}^{k+1}\right)-F\left(s_{i}, \phi_{i}^{k}\right)\right]+l_{i}^{i+1}\left(\phi^{k}\right) .
$$


We can use either (211) or (2.12) to compute a new approximation $\phi^{k+1}$ from $\phi^{k}$. Note that the only thing remained to be specified is how to compute $I_{i}^{i+1}\left(\phi^{k}\right)$.

The second key idea of the SDC method is to approximate $F\left(t, \phi^{k}\right)$ by its Lagrange interpolation polynomials $F_{p}\left(t, \phi^{k}\right)$ based on the Gauss-type points, and integrate $\int_{s_{i}}^{s_{i}+1} F_{p}\left(t, \phi^{k}\right) d t$ exactly. More precisely, we write

$$
F_{p}\left(t, \phi^{k}\right)=\sum_{j=1}^{p} F_{p}\left(t_{j}, \phi^{k}\right) \mathcal{L}_{j}^{p}(t),
$$

and approximate $I_{i}^{i+1}\left(\phi^{k}\right)$ by

$$
l_{i}^{i+1}\left(\phi^{k}\right) \approx \int_{s_{i}}^{s_{i+1}} F_{p}\left(t, \phi^{k}(t)\right) d t=\sum_{j=1}^{p} F_{p}\left(t_{j}, \phi^{k}\right) c_{i j j^{\prime}}^{p}
$$

where

$$
c_{i j}^{p}=\int_{s_{i}}^{s_{i+1}} \mathcal{L}_{j}^{p}(t) d t
$$

can be precomputed once and for all.

It is formally shown in [14] that the order of accuracy for the approximation $\phi^{k}$ is of order $\min (k+1,2 p-2)$ if $p$ Gauss-Radau points are used.

\subsection{Semi-implicit SDC schemes}

In general, it may not be good to use either the forward or backward Euler scheme for the correction Equation (2.4), as the system (2.1) may have terms with different time scales and/or different stiffness. Thus, it is plausible to treat some terms implicitly and other terms explicitly, leading to the so-called SISDC scheme (cf. [14, 30]). More precisely, assuming that we split $F(t, \phi(t))$ as $F_{l}(t, \phi(t))+F_{E}(t, \phi(t))$ and treat $F_{l}$ implicitly and $F_{E}$ explicitly. Then a first-order semi-implicit scheme for computing an initial approximation $\phi_{i}^{0}$ to Equation (2.1) is

$$
\phi_{i+1}^{0}=\phi_{i}^{0}+\delta t_{i} \cdot\left[F_{l}\left(s_{i+1}, \phi_{i+1}^{0}\right)+F_{E}\left(s_{i}, \phi_{i}^{0}\right)\right]
$$

Similarly, a semi-implicit scheme for (2.4) is

$$
\delta_{i+1}^{k}=\delta_{i}^{k}+\delta t_{i}\left[F_{l}\left(s_{i+1}, \phi_{i+1}^{k}+\delta_{i+1}^{k}\right)-F_{l}\left(s_{i+1}, \phi_{i+1}^{k}\right)+F_{E}\left(s_{i}, \phi_{i}^{k}+\delta_{i}^{k}\right)-F_{E}\left(s_{i}, \phi_{i}^{k}\right)\right]+\epsilon_{i+1}\left(\phi^{k}\right)-\epsilon_{i}\left(\phi^{k}\right),
$$

which can be rearranged as

$$
\phi_{i+1}^{k+1}=\phi_{i}^{k+1}+\delta t_{i}\left[F_{l}\left(s_{i+1}, \phi_{i+1}^{k+1}\right)-F_{l}\left(s_{i+1}, \phi_{i+1}^{k}\right)+F_{E}\left(s_{i}, \phi_{i}^{k+1}\right)-F_{E}\left(s_{i}, \phi_{i}^{k}\right)\right]+l_{i}^{i+1}\left(\phi^{k}\right) .
$$

In summary, given a system of ODEs (2.1), the only thing we need to specify for the previous SISDC scheme is the splitting $F=F_{l}+F_{E}$.

\section{Stabilized SISDC Schemes for the Allen-Cahn and Cahn-Hilliard Equations}

To apply the SISDC scheme described above to the Allen-Cahn and Cahn-Hilliard equations, the first step is to discretize them in space with a suitable spatial discretization to obtain a system of ODEs in the form of (2.1). Then, we need to construct a suitable splitting of $F=F_{I}+F_{E}$ to be used in (2.16).

\subsection{Spatial discretization}

Although the SISDC schemes presented previously can be combined with any consistent spatial discretization, we shall restrict ourselves to the spectral Galerkin method (cf. [31]), which has proven to be effective for interfacial problems modeled by Allen-Cahn and Cahn-Hilliard equations (cf. [21]).

For the readers' convenience, we shall present the 1D case in some detail; we refer to [31] for more detail on the multidimensional case.

We consider first the Legendre-Galerkin method for the Allen-Cahn equation. Let us denote

$$
X_{N}=\left\{u \in \mathbb{P}_{N}: \partial_{X} u( \pm 1)=0\right\}=\operatorname{span}\left\{\phi_{0}, \cdots, \phi_{N-2}\right\},
$$

with $\phi_{0}(x)=1$, and for $k \geq 1, \phi_{k}(x)=L_{k}(x)-b_{k} L_{k+2}(x)$, where $L_{k}(x)$ is the Legendre polynomial of $k$-th order, and

$$
b_{k}=\frac{k(k+1)}{(k+2)(k+3)}, k \geq 0 \text {. }
$$

It is easy to verify that $D_{i j}=\left(\phi_{j}^{\prime \prime}, \phi_{i}\right)=0$ for $i \neq j$, and $M_{i j}=\left(\phi_{j}, \phi_{i}\right)=0$ for $i \neq j, j \pm 2$. 
The Legendre-Galerkin method for (1.1) with (1.3) is to find $U_{N}(\cdot, t) \in X_{N}$ such that

$$
\left(\partial_{t} U_{N}, \phi_{k}\right)=\gamma\left(\partial_{x x} U_{N}-\frac{1}{\varepsilon^{2}} f\left(U_{N}\right), \phi_{k}\right), \quad k=0,1, \cdots, N-2
$$

Let us denote

$$
\begin{aligned}
U_{N}(x, t) & =\sum_{k=0}^{N-2} \tilde{u}_{k}(t) \phi_{k}(x), \quad \bar{u}=\left(\tilde{u}_{0}, \tilde{u}_{1}, \cdots, \tilde{u}_{N-2}\right)^{T}, \\
f(\bar{u})_{k}(t) & =-\left(f\left(U_{N}\right), \phi_{k}\right), \quad \bar{f}(\bar{u})=\left(f(\bar{u})_{0}, f(\bar{u})_{1}, \cdots, f(\bar{u})_{N-2}\right)^{T} .
\end{aligned}
$$

Then, (3.1) reduces to

$$
M \bar{u}^{\prime}(t)=\gamma\left[D \bar{u}(t)+\frac{1}{\varepsilon^{2}} \bar{f}(\bar{u}(t))\right]
$$

which can be written in the form of (2.1) as

$$
\bar{u}^{\prime}(t)=\gamma M^{-1}\left[D \bar{u}(t)+\frac{1}{\varepsilon^{2}} \bar{f}(\bar{u}(t))\right]:=F(t, \bar{u}(t)) .
$$

For the Cahn-Hilliard equation, we denote

$$
Y_{N}=\left\{u \in \mathbb{P}_{N}: \partial_{X} u( \pm 1)=\partial_{X}^{3} u( \pm 1)=0\right\}=\operatorname{span}\left\{\psi_{0}, \cdots, \psi_{N-4}\right\} .
$$

Setting $\psi_{k}(x)=L_{k}(x)+h_{k} L_{k+2}(x)+g_{k} L_{k+4}(x)$ with

$$
h_{k}=-\frac{2 k(k+1)(2 k+5)}{(2 k+7)\left(k^{2}+7 k+9\right)}, \quad g_{k}=\frac{k(k+1)(2 k+3)\left(k^{2}+3 k-1\right)}{(k+4)(k+5)(2 k+7)\left(k^{2}+7 k+9\right)}, k \geq 0 .
$$

It is easy to verify that $\tilde{D}_{i j}=-\left(\psi_{j}^{\prime \prime \prime \prime}, \psi_{i}\right)=0$ for $i \neq j, C_{i j}=\left(\psi_{j}^{\prime \prime}, \psi_{i}\right)=0$ for $i \neq j, j \pm 2$, and $\tilde{M}_{i j}=\left(\psi_{j}, \psi_{i}\right)=0$ for $i \neq j, j \pm 2, \pm 4$.

The Legendre-Galerkin method for (1.2) with (1.4) is to find $U_{N}(\cdot, t) \in Y_{N}$ such that

$$
\left(\partial_{t} U_{N}, \phi_{k}\right)=-\gamma\left(\partial_{x x x x} U_{N}-\frac{1}{\varepsilon^{2}} \partial_{x x} f\left(U_{N}\right), \psi_{k}\right), \quad k=0,1, \cdots, N-4
$$

Let us denote

$$
\begin{aligned}
U_{N}(x, t) & =\sum_{k=0}^{N-4} \tilde{u}_{k}(t) \psi_{k}(x), \quad \bar{u}=\left(\tilde{u}_{0}, \tilde{u}_{1}, \cdots, \tilde{u}_{N-4}\right)^{T}, \\
g(\bar{u})_{k}(t) & =\left(\partial_{x x} f\left(U_{N}\right), \psi_{k}\right), \quad \bar{g}(\bar{u})=\left(g(\bar{u})_{0}, g(\bar{u})_{1}, \cdots, g(\bar{u})_{N-4}\right)^{T} .
\end{aligned}
$$

Then, (3.5) reduces to

$$
\tilde{M} \bar{u}^{\prime}(t)=\gamma\left[\tilde{D} \bar{u}(t)+\frac{1}{\varepsilon^{2}} \bar{g}(\bar{u}(t))\right],
$$

which can be written in the form of (2.1) as

$$
\bar{u}^{\prime}(t)=\gamma \tilde{M}^{-1}\left[\tilde{D} \bar{u}(t)+\frac{1}{\varepsilon^{2}} \bar{g}(\bar{u}(t))\right]:=F(t, \bar{u}(t)) .
$$

Hence, we can directly apply the SISDC method to (3.4) and (3.8). The only ingredient remains to be specified is how to split $F(s, u)$ in (3.4) and (3.8) such that the basic scheme (2.16) is simple, easy to solve, and stable for large time steps.

\subsection{Stabilized semi-implicit scheme}

We now present stabilized semi-implicit schemes that determine the splitting of $F(s, \bar{u})$ in $(3.4)$ and (3.8).

Let us denote $E(u)=\|\nabla u\|^{2}+\frac{1}{\varepsilon^{2}} \int_{\Omega} \mathcal{F}(u) d x$. An important property of the Allen-Cahn Equation (1.1) is that its solution satisfies the following energy law:

$$
\frac{\partial}{\partial t} E(u)=-\gamma\left\|\Delta u-\frac{1}{\varepsilon^{2}} f(u)\right\|^{2} .
$$




$$
\frac{\partial}{\partial t} E(u)=-\gamma\left\|\nabla\left(\Delta u-\frac{1}{\varepsilon^{2}} f(u)\right)\right\|^{2} .
$$

Hence, it is highly desirable that the solution of a numerical scheme satisfy a corresponding discrete energy law. Such a numerical scheme is then said to be energy stable.

It can be shown that the usual first-order semi-implicit methods for Allen-Cahn and Cahn-Hilliard equations are energy stable under quite restrictive conditions $\Delta t=O\left(\varepsilon^{2}\right)$ and $\Delta t=O\left(\varepsilon^{4}\right)$, respectively, when $\varepsilon \ll 1$. Shen and Yang presented the following stabilized first-order semi-implicit methods and proved their stability in [21]. The stabilized first-order semi-implicit method for Allen-Cahn Equation (1.1) reads

$$
\left(\frac{1}{\delta t}+\frac{\gamma S}{\varepsilon^{2}}\right)\left(u^{k+1}-u^{k}\right)=\gamma\left[\Delta u^{k+1}-\frac{1}{\varepsilon^{2}} f\left(u^{k}\right)\right]
$$

whereas that for the Cahn-Hilliard Equation (1.2) is

$$
\frac{1}{\delta t}\left(u^{k+1}-u^{k}\right)=\gamma \Delta\left(-\Delta u^{k+1}+\frac{S}{\varepsilon^{2}}\left(u^{k+1}-u^{k}\right)+\frac{1}{\varepsilon^{2}} f\left(u^{k}\right)\right) .
$$

In the previous schemes, $S$ is a stabilizing parameter to be specified. The stabilizing term $\frac{\gamma S}{\varepsilon^{2}}\left(u^{k+1}-u^{k}\right)$ in $(3.11)\left(\right.$ resp. $\frac{\gamma S}{\varepsilon^{2}} \Delta\left(u^{k+1}-u^{k}\right)$ in (3.12)) introduces an extra consistency error that is of the same order as the error introduced by the explicit treatment of the nonlinear term (cf. [21]). Therefore, the stabilized semi-implicit schemes (3.11) and (3.12) are of the same order of accuracy, in terms of $\delta t$ and $\varepsilon$, as the standard first-order semi-implicit schemes, that is, with $S=0$ in (3.11) and (3.12). As for the stability, the following results are established in [21]:

Lemma 1

Let $S \geq \frac{L}{2}$ where $L=\max _{\phi \in \mathbb{R}}\left|f^{\prime}(\phi)\right|$. Then, the stabilized schemes (3.11) and (3.12) are unconditionally energy stable for any $\delta t$, that is, the solutions of (3.11) and (3.12) satisfy $E\left(u^{k+1}\right) \leq E\left(u^{k}\right)$ for all $k \geq 0$ and any $\delta t$.

Remark 1

Note that for $\mathcal{F}(\phi)$ being the double-well potential, we have $L=\max _{\phi \in \mathbb{R}}\left|\mathcal{F}^{\prime \prime}(\phi)\right|=\infty$ so the condition $S \geq \frac{L}{2}$ can not be satisfied. However, because it is well known that the Allen-Cahn equation satisfies the maximum principle, we can truncate $\mathcal{F}(u)$ to quadratic growth outside of the interval $[-M, M]$ without affecting the solution if the maximum norm of the initial condition $u_{0}$ is bounded by $M$. Although the Cahn-Hilliard equation does not satisfy the maximum principle, it has been shown in [32] that for a truncated potential $\mathcal{F}(u)$ with quadratic growth at infinities, the maximum norm of the solution for the Cahn-Hilliard equation is bounded. Therefore, it has been a common practice (cf. $[33,34]$ ) to replace the double-well potential by a truncated potential with quadratic growth at infinity so that $L$ is finite. In practice, if we assume $\max _{\phi \in \mathbb{R}}\left|u_{0}(\phi)\right|=1$, we can truncate $\mathcal{F}(\phi)$ outside of the interval $[-1,1]$ in such a way that $L=\max _{\phi \in \mathbb{R}}\left|\mathcal{F}^{\prime \prime}(\phi)\right|=2$.

At each time step, the stabilized scheme (3.11) (resp. (3.12)) leads to a Poisson (resp. biharmonic) type equation, which can be efficiently solved by the spectral Galerkin method (cf. [31]), so they are very good candidates for the basic scheme in the SDC approach. They will allow us to use large time steps with high-order accuracy in time, which is otherwise very difficult to achieve.

\subsection{Fully discretized SSISDC scheme}

We now combine all the ingredients presented previously to describe the SSISDC scheme with the Legendre-Galerkin approximation in space.

By using the notation in Subsection 3.1, the Legendre-Galerkin approximation for the stabilized semi-implicit scheme (3.11) for Allen-Cahn equation reads

$$
\left(\frac{1}{\delta t}+\frac{\gamma S}{\varepsilon^{2}}\right) M\left(\bar{u}^{k+1}-\bar{u}^{k}\right)=\gamma\left[D \bar{u}^{k+1}+\frac{1}{\varepsilon^{2}} \bar{f}\left(\bar{u}^{k}\right)\right],
$$

whereas the Legendre-Galerkin approximation for the stabilized semi-implicit scheme (3.12) Cahn-Hilliard equation is

$$
\frac{1}{\delta t} \tilde{M}\left(\bar{u}^{k+1}-\bar{u}^{k}\right)=\gamma \tilde{D} \bar{u}^{k+1}+\frac{\gamma S}{\varepsilon^{2}} C\left(\bar{u}^{k+1}-\bar{u}^{k}\right)+\frac{\gamma}{\varepsilon^{2}} \bar{g}\left(\bar{u}^{k}\right) .
$$

We shall use the previous schemes as the basic semi-implicit scheme in the SDC approach. More precisely, the scheme (3.13) corresponds to (2.16) with the splitting

$$
F_{l}(s, \bar{u})=\gamma\left(M^{-1} D \bar{u}-\frac{s}{\varepsilon^{2}} \bar{u}\right), \quad F_{E}(s, \bar{u})=\gamma\left(\frac{1}{\varepsilon^{2}} M^{-1} \bar{f}(\bar{u})+\frac{s}{\varepsilon^{2}} \bar{u}\right),
$$

whereas the scheme (3.14) corresponds to (2.16) with the splitting

$$
F_{l}(s, \bar{u})=\gamma M^{-1}\left(\tilde{D} \bar{u}+\frac{s}{\varepsilon^{2}} C \bar{u}\right), \quad F_{E}(s, \bar{u})=\gamma M^{-1}\left(\frac{1}{\varepsilon^{2}} \bar{g}(\bar{u})-\frac{s}{\varepsilon^{2}} C \bar{u}\right) .
$$




\section{Numerical Results}

In this section, we present some numerical results for Allen-Cahn and Cahn-Hilliard equations obtained by using the SSISDC method.

\subsection{Allen-Cahn equation}

We consider the one-dimensional Allen-Cahn Equation (1.1) with the homogeneous Neumann boundary conditions (1.3) and solve it by using the SSISDC method with the Legendre-Galerkin approximation described in the last section. We choose $N$, the number of modes in the spatial discretization, to be large enough that the spatial discretization error is negligible compared with the time discretization error.

We first investigate the stability issue. We fix $p=4$ and $J=2$, and start with a random initial condition with values in [-1, 1]. We define $\Delta t_{c}=\frac{T}{m}$ to be the largest possible time step, which allows stable numerical computation, that is, the numerical solution will blow up if $\delta t>\Delta t_{c}$. In Table l, we list the values of $\Delta t_{c}$ with $\gamma=0.1,0.01, \varepsilon=0.01,0.001$, and different stabilizing parameters of $S$. We observe that the stabilized scheme with $S=1$ or 2 is stable for virtually any $\Delta t$, whereas the non-stabilized scheme has a rather restrictive time step constraint.

Next, we investigate the effect of stabilizing parameter $S$ on the accuracy of the transient state as well as steady state solutions. We fix $p=4$ and $J=3$, and take $\gamma=0.01$ and $\varepsilon=0.01$ in (1.1). On the left of Figure 1, we observe that the stabilized scheme with $S=1,2$ leads to visually the same results at a transient time with a time step which is 50 times larger than the non-stabilized scheme (i.e., $S=0$ ), whereas for the steady state, the time step of the stabilized scheme with $S=1$ or 2 can be 1000 times larger while maintaining the same accuracy. We note that the time step of $10^{-4}$ is close to the maximum allowable time step for the non-stabilized scheme.

We now examine the order of accuracy as we increase the number of iteration. To fix the idea, we use the following parameters: $\gamma=0.01, \varepsilon=0.1, S=2$, and $N=127$, and we compute the $L^{\infty}$-norm of the residual defined in (2.7) at time $T=1$. We first take $p=4$ (4 Gauss-Radau lla points) and $J=1,2,3,4,5$. The decay rates of the residual with different number $J$ of iterations are shown in Figure 2. We observe that for $p$ and $J$, relatively small as in this example, the SSISDC scheme achieves the expected accuracy of order $\min (J+1,2 p-2)$.

\begin{tabular}{|c|c|c|c|}
\hline$\gamma$ & $\varepsilon$ & $S$ & $\Delta t_{c}$ \\
\hline$\gamma=0.1$ & $\varepsilon=0.01$ & $\begin{array}{l}S=0 \\
S=1 \\
S=2\end{array}$ & $\begin{array}{l}\Delta t_{c} \approx 3.0303 E-3 \\
\Delta t_{c}>10 \\
\Delta t_{c}>10\end{array}$ \\
\hline$\gamma=0.1$ & $\varepsilon=0.001$ & $\begin{array}{l}S=0 \\
S=1 \\
S=2\end{array}$ & $\begin{array}{l}\Delta t_{c} \approx 2.7027 E-5 \\
\Delta t_{c}>10 \\
\Delta t_{c}>10\end{array}$ \\
\hline$\gamma=0.01$ & $\varepsilon=0.001$ & $\begin{array}{l}S=0 \\
S=1 \\
S=2\end{array}$ & $\begin{array}{l}\Delta t_{c} \approx 2.7027 E-4 \\
\Delta t_{c}>10 \\
\Delta t_{c}>10\end{array}$ \\
\hline
\end{tabular}
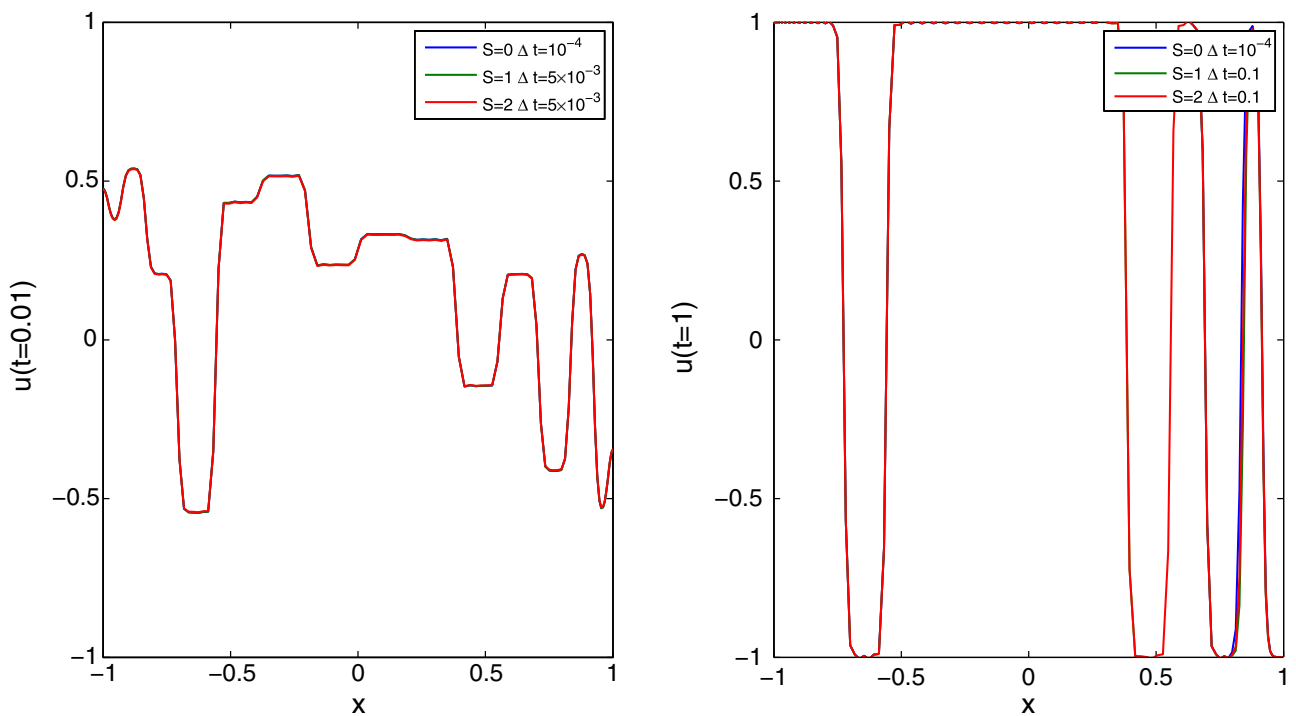

Figure 1. Comparison of the numerical solutions obtained by the SISDC and SSISDC method. Left: a transient state; Right: at the steady state. 


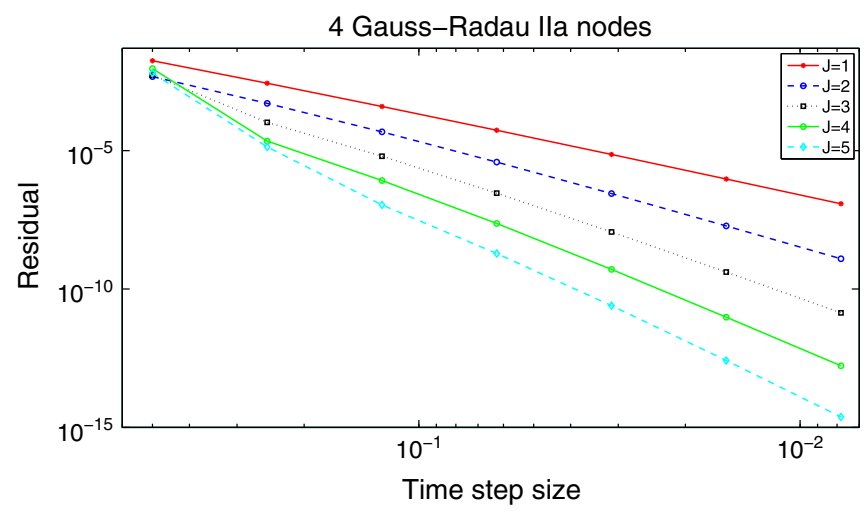

Figure 2. $L_{\infty}$ residual versus $\Delta t$ with $J=1, \cdots, 5(p=4)$.

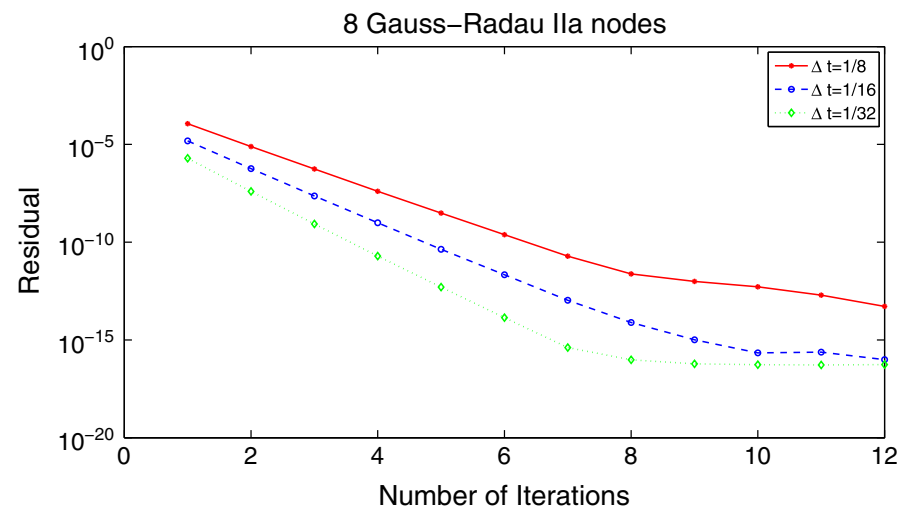

Figure 3. $L_{\infty}$ residual versus number of iterations $J$ with different $\Delta t(p=8)$.
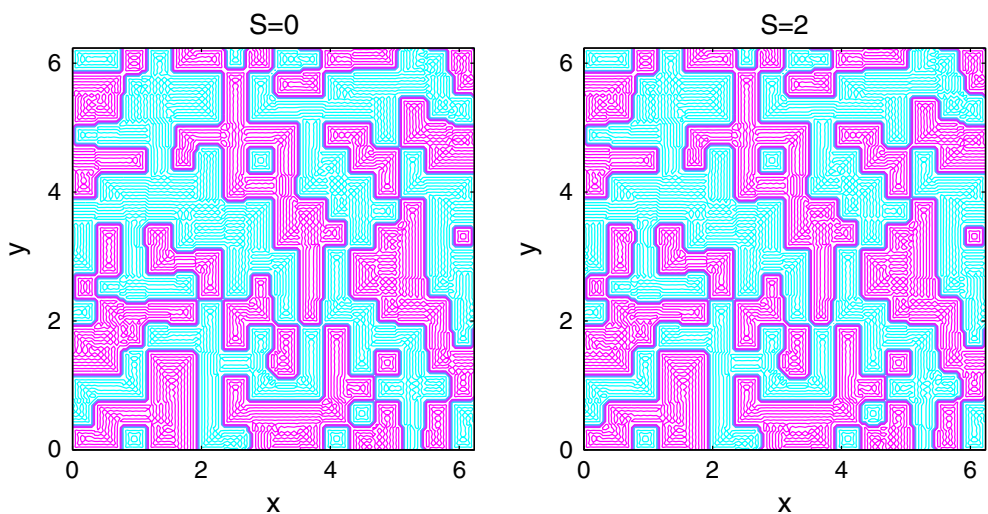

Figure 4. Comparison of the numerical solutions of the 2-D Allen-Cahn equation with $\gamma=0.01, \varepsilon=0.01$ at $T=10$. Left: by the non-stabilized SISDC scheme; Right: by the SSISDC scheme.

To examine the convergence behavior for lager numbers of iterations, we fix $p=8$ and take $\Delta t=\frac{1}{8}, \frac{1}{16}, \frac{1}{32}$, respectively. We plot in Figure 3 the residual versus $J$, the number of iterations. We observe that the errors decrease as the expected formal accuracy of order $\min (J+1,2 p-2)$ only for $J$ relatively small, and that the maximum order accuracy, $2 p-2$, does not seem to attainable. This type of order reduction is well known for stiff problems, and is consistent with the observation in [29], which suggests to use the KDC to accelerate the SDC method if very high-order of accuracy (i.e., $p$ large) is desired. However, for many PDEs as Allen-Cahn and Cahn-Hilliard equations, a time accuracy of 6th to 8th order (achievable with $p=4$ and 5, respectively) often suffices. In these cases, the simple and robust SDC method is more than adequate.

As the last example for the Allen-Cahn equation, we consider a two-dimensional domain $\Omega=[0,2 \pi]^{2}$ with periodic boundary conditions. We start with a random initial data and integrated the Allen-Cahn equation with $\gamma=0.01, \varepsilon=0.01$ to $T=10$. The Fourier spectral method with 128 Fourier modes in each direction is used. Figure 4 shows the solution comparisons at $T=10$ using the SISDC scheme with $\Delta t=10^{-3}$ and the SSISDC scheme with $S=2$ and $\Delta t=0.1$. The two results are visually indistinguishable. 


\subsection{Cahn-Hilliard Equation}

We now consider the Cahn-Hilliard Equation (1.2) with the homogeneous Neumann boundary conditions (1.4), and present a same set of numerical results as for the Allen-Cahn equation.

The results in Table II are similar to those in Table I, except that the time step for the non-stabilized scheme becomes much more restrictive. Therefore, it is extremely beneficial to use a stabilized scheme that is unconditionally stable.

Next, we investigate the effect of the stabilizing term on the accuracy. We first compute the residuals with different stabilizing parameters with varying $\Delta t$ by fixing $p=4, J=3, \varepsilon=1$, and $\gamma=0.01$. The results are shown in Figure 5 which shows that the residuals are almost the same with stabilizing parameters $S=0,1,2$ even with relatively large time steps.

\begin{tabular}{|c|c|c|c|}
\hline$\gamma$ & $\varepsilon$ & $S$ & $\Delta t_{c}$ \\
\hline$\gamma=0.1$ & $\varepsilon=0.1$ & $\begin{array}{l}S=0 \\
S=1 \\
S=2\end{array}$ & $\begin{array}{l}\Delta t_{c} \approx 0.009 \\
\Delta t_{c}>1 \\
\Delta t_{c}>1\end{array}$ \\
\hline$\gamma=0.1$ & $\varepsilon=0.01$ & $\begin{array}{l}S=0 \\
S=1 \\
S=2\end{array}$ & $\begin{array}{l}\Delta t_{c} \approx 9 E-7 \\
\Delta t_{c}>1 \\
\Delta t_{c}>1\end{array}$ \\
\hline$\gamma=0.01$ & $\varepsilon=0.01$ & $\begin{array}{l}S=0 \\
S=1 \\
S=2\end{array}$ & $\begin{array}{l}\Delta t_{c} \approx 9 E-6 \\
\Delta t_{c}>1 \\
\Delta t_{c}>1\end{array}$ \\
\hline
\end{tabular}

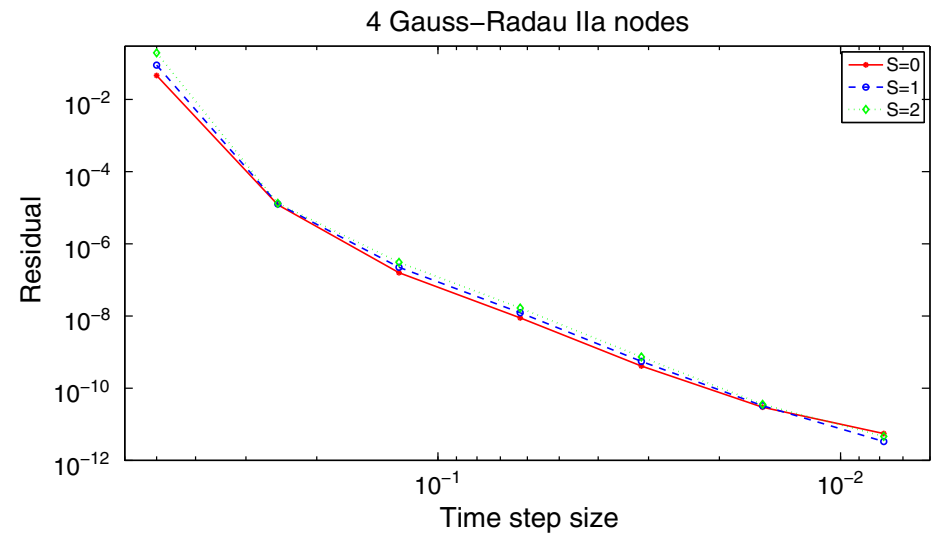

Figure 5. $L_{\infty}$ residual versus $\Delta t$ with different stabilization parameters.
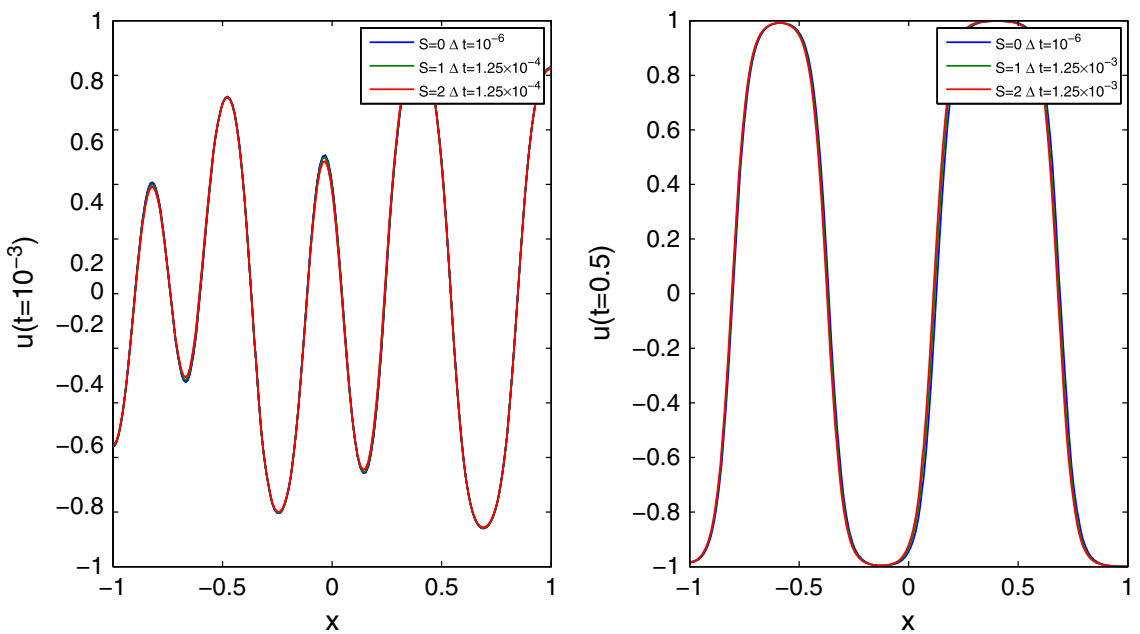

Figure 6. Comparison of the numerical solutions obtained by the SISDC and SSISDC method for the Cahn-Hilliard equation. Left: at a transient time; Right: at the steady state. 


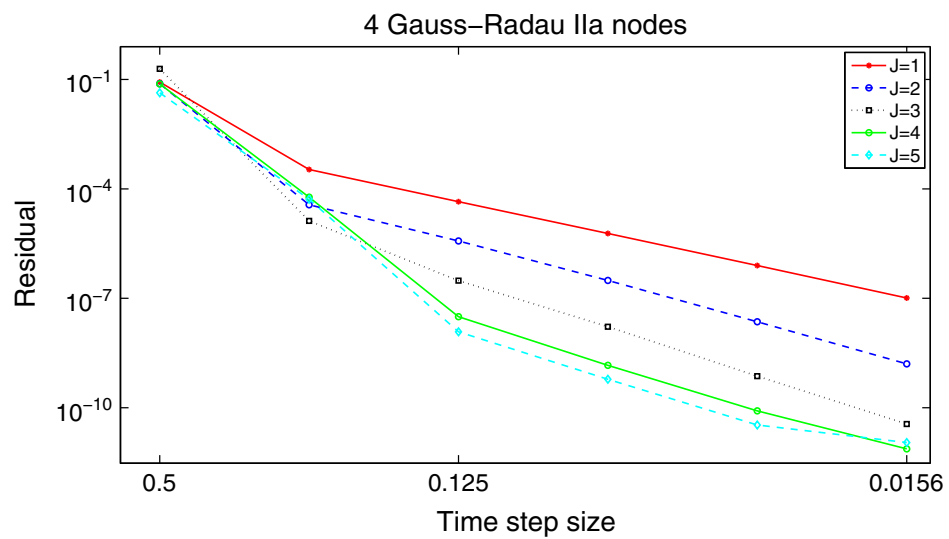

Figure 7. $L_{\infty}$ residual versus $\Delta t$ with $J=1, \cdots, 5(p=4)$.

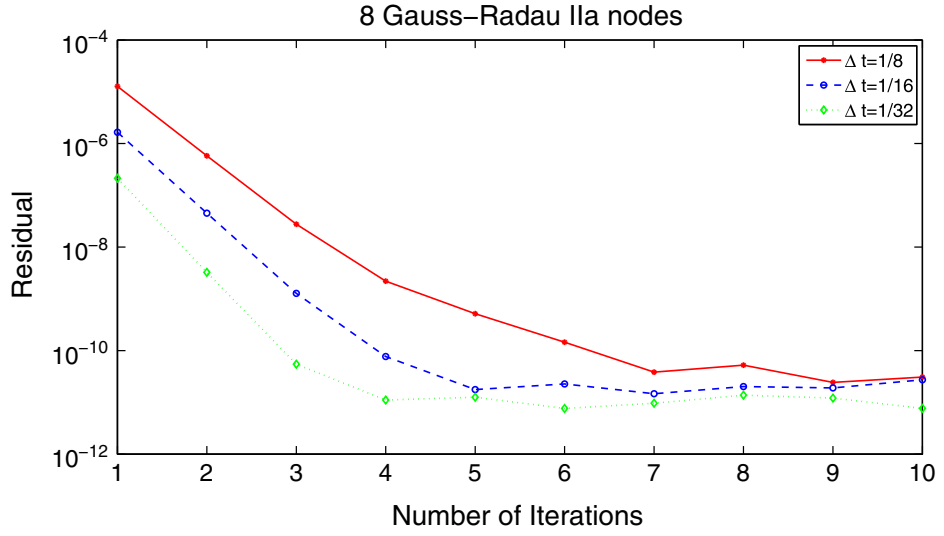

Figure 8. $L_{\infty}$ residual versus the number of iterations $J$ with different $\Delta t(p=8)$.
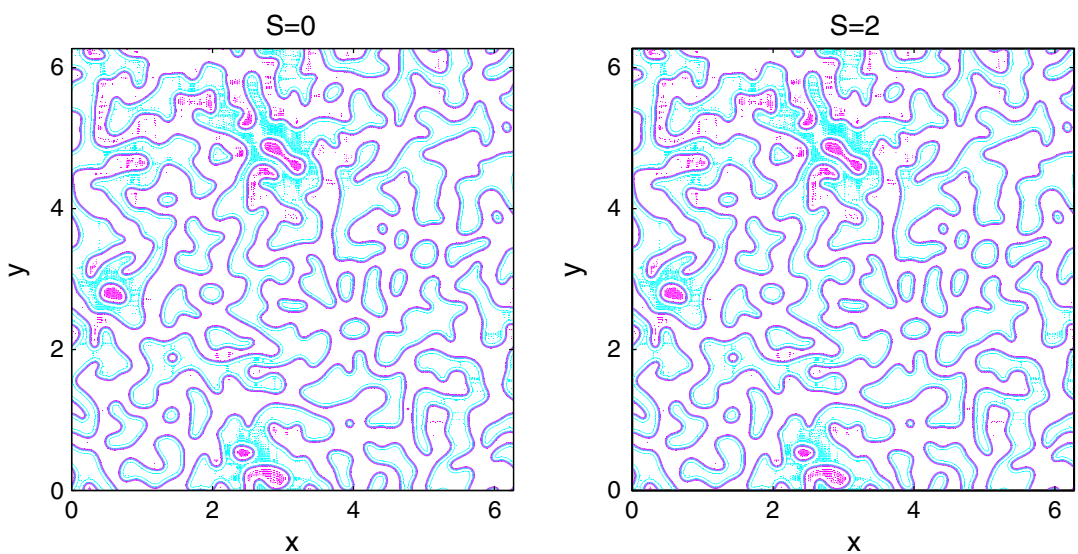

Figure 9. Comparison of the numerical solutions of the 2D Cahn-Hilliard equation with $\gamma=10^{-4}, \varepsilon=0.01$ at $T=10$. Left: by the non-stabilized SISDC scheme; Right: by the SSISDC scheme.

We then fix $p=4, J=3$ and take $\gamma=0.1$ and $\varepsilon=0.05$ in (1.2). On the left (resp. right) of Figure 6, we plot the solutions at a transient time (resp. the steady state) obtained by using the non-stabilized and stabilized schemes. We observe that for the transient time (resp. the steady state), the time step of the stabilized scheme can be 125 (resp. 1250) times larger than that of the non-stabilized scheme with no visible difference in the numerical solutions.

In the next two experiments, we fix $\gamma=0.01, \varepsilon=1$ in (1.2), and examine the order of accuracy of the SSISDC scheme with different number of iterations. As for the Allen-Cahn equation, we first take $p=4$ with $J$ up to 5 . Figure 7 shows how the residual decays as $J$ increases with different time step sizes. It is observed that the scheme achieves the expected formal accuracy of order min $(J+1,2 p-2)$ only for $J=1,2,3$. As we further increase $J$, the order of convergence does not behave like $\min (J+1,2 p-2)$. Similar situation is observed for $p=8$ (cf. Figure 8). The order reduction of the SSISDC method for the Cahn-Hilliard equation appears to be more severe than that 
for the Allen-Cahn equation, but this is not surprising because the ODE system from the semi-discretization of Cahn-Hilliard equation is much more stiff than that of the Allen-Cahn equation.

As the final example for the Cahn-Hilliard equation, we solve the Cahn-Hilliard Equation (1.2) with $\gamma=10^{-4}$, $\varepsilon=0.01$ and periodic boundary conditions in the domain $\Omega=[0,2 \pi]^{2}$ using the non-stabilized SISDC scheme and the SSISDC scheme with a Fourier discretization in space. We start with a random initial condition and use 512 Fourier modes in each direction. We plot Figure 9 the contour lines of the solution at $T=10$ by using the non-stabilized SISDC scheme (i.e., $S=0$ ) with $\Delta t=10^{-5}$ and the SSISDC scheme with $S=2$ and $\Delta t=0.05$. No visible difference is observable.

\section{Concluding remarks}

We presented SSISDC methods for the time discretization of Allen-Cahn and Cahn-Hilliard equations. Although these methods can be coupled with any consistent spatial discretization, we used Legendre-Galerkin method for nonperiodic boundary conditions and Fourier-Galerkin method for periodic boundary conditions. The combined algorithms are unconditionally stable, lead to simple linear system to solve at each iteration, and can achieve high-order time accuracy with a few iterations in each time step.

We presented ample numerical results to demonstrate the effectiveness of the SSISDC methods. It is observed that the SSISDC methods can achieve a relatively high-order accuracy in time with just a few SDC iterations. However, due to the stiffness in the ODE systems from the semi-discretizations of the Allen-Cahn and the Cahn-Hilliard equations, the actual order of accuracy is usually less than the expected formal accuracy of order $\min (J+1,2 p-2)$ for large $J$ and $p$. If very high order of accuracy is desired, one can use instead the so-called KDC method. However, it usually takes a quite large number of iterations to reach the maximum order of accuracy of $2 p-2$.

In conclusion, the SSISDC methods are very effective for solving Allen-Cahn and Cahn-Hilliard equations. In particular, they allow us to use much larger time steps to obtain moderately high-order of accuracy with only a few iterations at each time step. They can also be generalized to deal with more general gradient flows, and can be used as a major ingredient for developing highly efficient and accurate numerical schemes for other systems involving Allen-Cahn and Cahn-Hilliard equations such as the phase-field model for multiphase incompressible flows.

\section{Acknowledgements}

Fei Liu is partially supported by the Chinese Universities Scientific Fund No. 2010QNA3019. Jie Shen is partially supported by the grant of National Natural Science Foundation of China No. 91130002.

\section{References}

1. Allen SM, Cahn JW. A microscopic theory for antiphase boundary motion and its application to antiphase domain coarsening. Acta Metallurgica et Materialia 1979; 27:1085-1095.

2. Cahn JW, Hilliard JE. Free energy of a nonuniform system. I. Interfacial free energy. Journal of Chemical Physics 1958; 28:258.

3. Elliott CM, French DA. Numerical studies of the Cahn-Hilliard equation for phase separation. IMA Journal of Applied Mathematics 1987; 38(2):97-128.

4. Du Q, Nicolaides RA. Numerical analysis of a continuum model of phase transition. SIAM Journal on Numerical Analysis 1991; 28(5):1310-1322.

5. Elliott CM, Garcke H. On the Cahn-Hilliard equation with degenerate mobility. SIAM Journal on Mathematical Analysis 1996; 27:404-423.

6. Zhu J, Chen LQ, Shen J, Tikare V. Coarsening kinetics from a variable-mobility Cahn-Hilliard equation: application of semi-implicit Fourier spectral method. Physical Review E 1999; 60:3564-3572.

7. Gottlieb D, Orszag SA. Numerical Analysis of Spectral Methods: Theory and Applications. SIAM-CBMS: Philadelphia, 1977.

8. Boyd JP. Chebyshev and Fourier Spectral Methods, Second edition. Dover Publications Inc.: Mineola, NY, 2001.

9. Karniadakis GE, Sherwin SJ. Spectral/hp Element Methods for Computational Fluid Dynamics, Second edition, Numerical Mathematics and Scientific Computation. Oxford University Press: New York, 2005.

10. Canuto C, Hussaini MY, Quarteroni A, Zang TA. Spectral Methods, Scientific Computation. Springer-Verlag: Berlin, 2006. Fundamentals in single domains.

11. Shen J, Tang T, Wang L-L. Spectral Methods: Algorithms, Analysis and Applications, Springer Series in Computational Mathematics, Vol. 41. Springer: Heidelberg, 2011.

12. Tal-Ezer H. Spectral methods in time for parabolic problems. SIAM Journal on Numerical Analysis 1989; 26(1):1-11.

13. Bar-Yoseph P, Moses E, Zrahia U, Yarin AL. Space-time spectral element methods for one-dimensional nonlinear advection-diffusion problems. Journal of Computational Physics 1995; 119(1):62-74.

14. Dutt A, Greengard L, Rokhlin V. Spectral deferred correction methods for ordinary differential equations. BIT 2000; 40(2):241-266.

15. Shen J, Wang L-L. Analysis of a spectral-Galerkin approximation to the Helmholtz equation in exterior domains. Applied Numerical Mathematics 2007; 57:710-720.

16. Huang J, Jia J, Minion M. Arbitrary order Krylov deferred correction methods for differential algebraic equations. Journal of Computational Physics 2007; $221(2): 739-760$.

17. Layton AT, Minion ML. Implications of the choice of predictors for semi-implicit Picard integral deferred correction methods. Communications in Applied Mathathematics and Computer Sciece 2007; 2:1-34.

18. Jia J, Huang J. Krylov deferred correction accelerated method of lines transpose for parabolic problems. Journal of Computational Physics 2008; 227(3):1739-1753.

19. Hansen AC, Strain J. On the order of deferred correction. Applied Numerical Mathematics 2011; 61(8):961-973.

20. Eyre DJ. Unconditionally gradient stable time marching the Cahn-Hilliard equation. In Computational and Mathematical Models of Microstructural Evolution (San Francisco, CA, 1998), Vol. 529, Mater. Res. Soc. Sympos. Proc. MRS: Warrendale, PA, 1998; 39-46. 
21. Shen J, Yang X. Numerical approximations of Allen-Cahn and Cahn-Hilliard equations. Discrete and Continuous Dynamical Systems 2010; 28(4): 1669-1691.

22. Li B, Liu J-G. Thin film epitaxy with or without slope selection. European Journal on Applied Mathematics 2003; 14(6):713-743.

23. Evans JW, Thiel PA, Bartelt MC. Morphological evolution during epitaxial thin film growth: formation of 2D islands and 3D mounds. Surface Science Reports 2006; 61:1-128.

24. Wang C, Wang X, Wise SM. Unconditionally stable schemes for equations of thin film epitaxy. Discrete and Continuous Dynamical Systems 2010; 28(1):405-423.

25. Taylor JE, Cahn JW. Diffuse interfaces with sharp corners and facets: phase field models with strongly anisotropic surfaces. Physica $D$ 1998; 112(3-4):381-411.

26. Wise S, Kim J, Lowengrub J. Solving the regularized, strongly anisotropic Cahn-Hilliard equation by an adaptive nonlinear multigrid method. Journal of Computational Physics 2007; 226(1):414-446.

27. Torabi S, Lowengrub J, Voigt A, Wise S. A new phase-field model for strongly anisotropic systems. Proceedings of the Royal Society A: Mathematical, Physical and Engineering Science 2009; 465(2105):1337-1359.

28. Liu C, Shen J. A phase field model for the mixture of two incompressible fluids and its approximation by a Fourier-spectral method. Physica D 2003; 179(3-4):211-228.

29. Huang J, Jia J, Minion M. Accelerating the convergence of spectral deferred correction methods. Journal of Computational Physics 2006; 214(2):633-656.

30. Minion ML. Semi-implicit spectral deferred correction methods for ordinary differential equations. Communications in Mathematical Sciences 2003; 1:471-500

31. Shen J. Efficient spectral-Galerkin method I. Direct solvers for second- and fourth-order equations by using Legendre polynomials. SIAM Journal of Scientific Computing 1994; 15:1489-1505.

32. Caffarelli LA, Muler NE. An $L^{\infty}$ bound for solutions of the Cahn-Hilliard equation. Archive for Rational Mechanics and Analysis 1995; 133(2):129-144.

33. Kessler D, Nochetto RH, Schmidt A. A posteriori error control for the Allen-Cahn problem: circumventing Gronwall's inequality. M2AN Mathematical Modelling and Numerical Analysis 2004; 38(1):129-142.

34. Condette N, Melcher C, Süli E. Spectral approximation of pattern-forming nonlinear evolution equations with double-well potentials of quadratic growth. Mathematics of Computation 2011; 80(273):205-223. 